\title{
Performance Analysis of an Adaptive Queue-Aware Random Access Scheme with Random Traffic
}

Ioannis Dimitriou and Nikolaos Pappas

\section{Conference article}

Cite this conference article as:

Dimitriou, I., Pappas, N. Performance Analysis of an Adaptive Queue-Aware Random Access Scheme with Random Traffic, In 2018 IEEE International Conference on Communications (ICC) Proceedings Kansas City, MO, USA 20- 24 May 2018: IEEE Communications Society; 2018, pp. 1-6. ISBN: 9781538631805

DOI: https:// doi.org/ 10.1109/ICC.2018.8422688

Copyright: IEEE Communications Society

Publisher's website: https:// ieeexplore.ieee.org/

The self-archived postprint version of this conference article is available at Linköping University Institutional Repository (DiVA):

http:// urn.kb.se/ resolve?urn=urn:nbn:se:liu:diva-144827 


\title{
Performance Analysis of an Adaptive Queue-Aware Random Access Scheme with Random Traffic
}

\author{
Ioannis Dimitriou ${ }^{1}$, Nikolaos Pappas ${ }^{2}$ \\ ${ }^{1}$ Department of Mathematics, University of Patras, 26500, Patras, Greece \\ ${ }^{2}$ Department of Science and Technology, Linköping University, Campus Norrköping, Sweden \\ Email: idimit@math.upatras.gr, nikolaos.pappas@liu.se
}

\begin{abstract}
In this work, we consider an adaptive two-user random access scheme with multipacket reception (MPR) capabilities. A user adapts its transmission characteristics based on the status of the other user. The users have external random traffic stored in their queues. We derive the stable throughput region of the system and the convexity conditions of this region. We derive analytically the queueing delay by formulating and solving a Riemann-Hilbert boundary value problem. Finally, we evaluate numerically the presented theoretical results.
\end{abstract}

\section{INTRODUCTION}

In this work, we study a two-user random access wireless network with multi-packet reception (MPR) capabilities. Random access has re-gained interest due to the increased number of communicating devices in $5 \mathrm{G}$ networks, and the need of massive uncoordinated access in large networks [1], [2]. Random access and alternatives and their effect on the operation of LTE and LTE-A are studied in [1], [3], [4]. The implications of random access in physical layer has been studied in [5], [6], [7].

When the traffic in a network is bursty, the characterization of the stability, i.e., stable throughput, region is of major importance. The exact characterization of the stability region is a quite difficult task due to the interaction among the queues. Thus, the individual departure rates of the queues cannot be computed separately without knowing the stationary distribution of the joint queue length process [8]. The vast majority of previous works on random access has focused on small-sized networks and only bounds or approximations are known for the networks with larger number of sources [6], [8], [9], [10], [11], [12], [13].

On the other hand, delay is an important metric, which recently received considerable attention due to the rapid growth on supporting real-time applications. However, the characterization of the delay even in small random-access networks is a rather challenging task. Delay analysis of random access networks was studied in [11], [14], [15], [16]. In [14] an asymmetric two-user network with collision channel was studied and the expressions for the average delay were obtained, while in [6], [11], a symmetric two-user network with MPR capabilities was considered.

This work has been partially supported by the EU project DECADE under Grant H2020-MSCA-2014-RISE: 645705, the European Unions Horizon 2020 research and innovation programme. This work has been partially supported by CENIIT.

\section{A. Contributions}

We consider an asymmetric two-user random access wireless network with a common destination and MPR capabilities. We employ a queue-aware transmission scheme, under which, the nodes adapt their transmission probabilities based on the status of the queue of the other nodes. This adaptation increases the chances of a successful packet transmission. We derive the stability region of the network and we obtain the conditions under which the stability region is a convex set. Furthermore, we provide a concrete mathematical analysis in order to study the queueing delay at user nodes in terms of the solution of a Riemann-Hilbert boundary value problem (e.g., [14], [17], [18], [19], [20]). The analysis is rather complicated and novel. Note that the fundamental problem of characterizing the delay in a general random access network with arbitrary number of users still remains an open problem, due the high level of interaction among the queues. However, our analysis presented in this work provides a building block towards the generalization to larger topologies. For the symmetric case we obtain a lower and an upper bound for the average delay without the need of solving a boundary value problem. These bounds as it is seen in the numerical results appear to be tight. The exact expressions for the average delay are obtained for the channel model with capture effect.

\section{MODEL DESCRIPTION}

We consider a slotted random access system consisting of two users communicating with a common receiver as presented in Fig. 1. In this work the terms users and nodes are used interchangeably. Each user has an infinite capacity buffer, in which stores the arriving packets. The packets have equal length and the time is divided into slots corresponding to the transmission time of a packet. Let $\left\{A_{k, n}\right\}_{n \in \mathbf{N}}$ be a sequence of independent and identically distributed random variables where $A_{k, n}$ is the number of packets arriving at the user node $k, k=1,2$, within the time interval $(n, n+1]$, with $E\left(A_{k, n}\right)=\lambda_{k}<\infty$. We denote by $N_{k, n}$ the number of packets stored at node $k$, at the beginning of slot $n$, and $N_{k}=\lim _{n \rightarrow \infty} N_{k, n}, k=1,2$.

At the beginning of each slot, there is a possibility for the node $k, k=1,2$, to transmit a packet to the receiver if its queue is not empty. Note that, since the destination node has MPR capability, more than one concurrent transmission may occur without having a collision. 


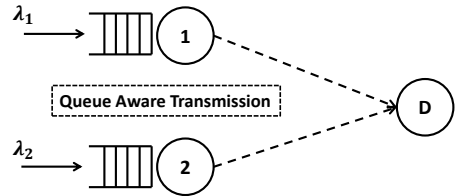

Fig. 1. The two-user random access wireless network with random arrivals.

In this work, we consider the following queue-aware transmission policy. If both nodes are non-empty, then node $k$, $k=1,2$, transmits a packet with probability $\alpha_{k}$ independently and $\bar{\alpha}_{k}=1-\alpha_{k}$ is the probability that node $k$ does not make a transmission. If node 1 (resp. 2) is the only non-empty, it transmits a packet with probability $\alpha_{k}^{*}\left(\bar{\alpha}_{k}^{*}=1-\alpha_{k}^{*}\right)$. We consider the general case for $\alpha_{k}^{*}$ instead of assuming directly $\alpha_{k}^{*}=1$. This, can handle cases where the node cannot transmit with probability one even if the other node is silent, e.g., due to energy limitations. Here, we do not consider specific reasons when this case can appear but our intention is to keep the proposed analysis as general as possible. Note that in our case, a node is aware about the state of its neighbor. In a shared access network, it is practical to assume some minimum exchanging information of one bit in this case.

\section{A. Physical Layer Model}

The MPR channel model used in this paper is a generalized form of the packet erasure model. In a wireless environment, a packet can be decoded correctly by the receiver if the received SINR exceeds a certain threshold. Suppose that given a set $T$ of nodes transmitting in the same time slot, let $P_{r x}(i, j)$ be the received power at node $j$ (when $i$ transmits). The SINR received by node $j$ is $\operatorname{SINR}(i, j)=\frac{P_{r x}(i, j)}{n_{j}+\sum_{k \in T-\{i\}} P_{r x}(k, j)}$, where $n_{j}$ denotes the noise power at receiver $j$. We assume that a packet transmitted by $i$ is successfully received by $j$ if and only if $\operatorname{SINR}(i, j) \geq \gamma_{i}$, where $\gamma_{i}$ is the SINR threshold. The wireless channel is subject to fading; let $P_{t x}(i)$ be the transmitting power at node $i$ and $r(i, j)$ be the distance between $i$ and $j$. The power received by $j$ when $i$ transmits is $P_{r x}(i, j)=A(i, j) g(i, j)$ where $A(i, j)$ is a random variable representing small-scale channel fading. We assume that fading is constant during a time slot and independently varying from time slot to time slot. Under Rayleigh fading, it is known that $A(i, j)$ is exponentially distributed. The received power factor $g(i, j)=P_{t x}(i)(r(i, j))^{-h}$ where $h$ is the path loss exponent with typical values between 2 and 6 . In this study, we consider one common destination for both nodes, thus, $j$ denotes the common destination and we can write $\operatorname{SINR}(i, j)=S I N R_{i}$. The expression for the success probability of link $i, j$ when the transmitting nodes are in $T$ denoted by $P_{s}(i, T)$ can be found in the literature; see [21].

Next, we will define for convenience some conditional probabilities based on the $P_{s}(i, T)$, a similar approach can be found in [11], [6]. We define $P_{1 /\{1,2\}}$ the probability that when both nodes 1 and 2 are transmitting only the transmission from node 1 is successful. Then $P_{1 /\{1,2\}} \triangleq$ $\operatorname{Pr}\left(S I N R_{1} \geq \gamma_{1}, S I N R_{2}<\gamma_{2}\right)$. Similarly we can define $P_{2 /\{1,2\}}$. The $P_{1,2 /\{1,2\}}$ is the probability that both packets transmitted by nodes 1 and 2 are transmitted success- fully, then $P_{1,2 /\{1,2\}} \triangleq \operatorname{Pr}\left(S I N R_{1} \geq \gamma_{1}, S I N R_{2} \geq \gamma_{2}\right)$. Then we have $P_{s}(1,\{1,2\})=P_{1 /\{1,2\}}+P_{1,2 /\{1,2\}}=$ $\operatorname{Pr}\left(S I N R_{1} \geq \gamma_{1}\right)=\operatorname{Pr}\left(S I N R_{1} \geq \gamma_{1}, S I N R_{2}<\gamma_{2}\right)+$ $\operatorname{Pr}\left(S I N R_{1} \geq \gamma_{1}, S I N R_{2} \geq \gamma_{2}\right)$.

The term $P_{0 /\{1,2\}} \triangleq \operatorname{Pr}\left(S I N R_{1}<\gamma_{1}, S I N R_{2}<\gamma_{2}\right)$ is the probability where both packets fail to reach the destination when both nodes 1 and 2 are transmitting, then $P_{0 /\{1,2\}}=1-$ $P_{1 /\{1,2\}}-P_{2 /\{1,2\}}-P_{1,2 /\{1,2\}}$. Note that $P_{i /\{i\}}=P_{s}(i,\{i\})$ is the success probability of node $i$ when only $i$-th node transmits but the other one is active (i.e., there are packets stored in its buffer), we denote $P_{0 /\{i\}}=1-P_{s}(i,\{i\})$ the outage probability respectively.

We employ a simple power control policy, under which a node adjusts its transmission parameters when the other node has an empty queue (i.e is inactive). Thus, denote by $\tilde{P}_{i /\{i\}}$ (resp. $\tilde{P}_{0 /\{i\}}$ ) the success (resp. outage) probability of node $i$ when the other node is inactive. Under such a scenario it is reasonable to assume that $\tilde{P}_{i /\{i\}} \geq P_{i /\{i\}}$.

In case of an unsuccessful transmission the packet has to be re-transmitted later. We assume that the receiver gives an instantaneous error-free feedback (ACK) of all the packets that were successful in a slot at the end of the slot. The successfully transmitted packets are removed, while the packets that were not successfully transmitted are retained.

\section{Stability Region}

In this section, we derive the stability region, i.e., the region of values for $\lambda_{k}, k=1,2$, for which our system is stable.

Theorem III.1. The stability region $\mathcal{R}$ for a fixed transmission probability vector $\mathbf{p}:=\left[\alpha_{1}, \alpha_{1}^{*}, \alpha_{2}, \alpha_{2}^{*}\right]$ is given by $\mathcal{R}=\mathcal{R}_{1} \cup$ $\mathcal{R}_{2}$ where

$$
\begin{aligned}
& \mathcal{R}_{1}=\left\{\left(\lambda_{1}, \lambda_{2}\right): \lambda_{1}<\alpha_{1}^{*} \tilde{P}_{1 /\{1\}}+\widehat{d}_{1} \frac{\lambda_{2}}{\alpha_{2} \widehat{\alpha}_{1}}, \lambda_{2}<\alpha_{2} \widehat{\alpha}_{1}\right\} \\
& \mathcal{R}_{2}=\left\{\left(\lambda_{1}, \lambda_{2}\right): \lambda_{2}<\alpha_{2}^{*} \tilde{P}_{2 /\{2\}}+\widehat{d}_{2} \frac{\lambda_{1}}{\alpha_{1} \widehat{\alpha}_{2}}, \lambda_{1}<\alpha_{1} \widehat{\alpha}_{2}\right\}
\end{aligned}
$$

where $\widehat{d}_{k}=d_{k}+\alpha_{1} \alpha_{2} P_{1,2 /\{1,2\}}$ for $k=1,2, d_{1}=$ $\alpha_{1}\left(\bar{\alpha}_{2} P_{1 /\{1\}}+\alpha_{2} P_{1 /\{1,2\}}\right)-\alpha_{1}^{*} \tilde{P}_{1 /\{1\}}, d_{2}=\alpha_{2}\left(\bar{\alpha}_{1} P_{2 /\{2\}}+\right.$ $\left.\alpha_{1} P_{2 /\{1,2\}}\right)-\alpha_{2}^{*} \tilde{P}_{2 /\{2\}}, \widehat{\alpha}_{1}=\bar{\alpha}_{1} P_{2 /\{2\}}+\alpha_{1}\left(P_{2 /\{1,2\}}+\right.$ $\left.P_{1,2 /\{1,2\}}\right), \widehat{\alpha}_{2}=\bar{\alpha}_{2} P_{1 /\{1\}}+\alpha_{2}\left(P_{1 /\{1,2\}}+P_{1,2 /\{1,2\}}\right)$. The stability region $\mathcal{R}$, is depicted in Fig. 2.

Proof. To determine the stability region of our system we apply the stochastic dominance technique [8], i.e. we construct hypothetical dominant systems, where one source when is empty, transmits a dummy packet, while the other queue transmits according to its traffic, as in the original system. Under this approach, we consider the first, and second-dominant systems. In the first (second) dominant system, whenever the queue of user one (two), empties, transmits a dummy packet.

Then, in first dominant system, node 1 never empties, and hence, node 2 sees a constant service rate, while the service rate of node 1 depends on the state of node 2 . We proceed with the queue at node 1 . The service rate of the first node is given by

$$
\begin{aligned}
& \mu_{1}=\operatorname{Pr}\left(N_{2} \neq 0\right)\left[\alpha_{1} \bar{\alpha}_{2} P_{1 /\{1\}}+\alpha_{1} \alpha_{2}\right. \\
& \left.\times\left(P_{1 /\{1,2\}}+P_{1,2 /\{1,2\}}\right)\right]+\operatorname{Pr}\left(N_{2}=0\right) \alpha_{1}^{*} \tilde{P}_{1 / 1},
\end{aligned}
$$


while the service rate of the second user is given by,

$$
\mu_{2}=\alpha_{2} \bar{\alpha}_{1} P_{2 /\{2\}}+\alpha_{2} \alpha_{1}\left(P_{2 /\{1,2\}}+P_{1,2 /\{1,2\}}\right) .
$$

By applying Loyne's criterion, the second node is stable if and only if the average arrival rate is less that the average service rate, $\lambda_{2}<\alpha_{2} \bar{\alpha}_{1} P_{2 /\{2\}}+\alpha_{2} \alpha_{1}\left(P_{2 /\{1,2\}}+P_{1,2 /\{1,2\}}\right)$. We can obtain the probability that the second node is empty and is given by $\operatorname{Pr}\left(N_{2}=0\right)=1-\frac{\lambda_{2}}{\mu_{2}}$. After replacing $\operatorname{Pr}\left(N_{2}=0\right)$ into (3), and applying Loynes criterion we obtain the stability condition for the first node. Then, we have the stability region $\mathcal{R}_{1}$ given by (1). Note that the expression in (1) is given in a more compact form that it will be useful in the next sections. Similarly, we can obtain the stability region for the second dominant system $\mathcal{R}_{2}$ given in (2), the proof is omitted due to space limitations. For a detailed treatment of dominant systems please refer to [8].

The stability conditions obtained by the stochastic dominance technique are not only sufficient but also necessary for the stability of the original system by introducing the indistinguishability argument [8]. Based on the construction of the dominant system, the queue sizes in the dominant system are always greater than those in the original system, provided they are both initialized to the same value and the arrivals are identical in both systems. Therefore, given $\lambda_{2}<\mu_{2}$, if for some $\lambda_{1}$, the queue at the first user is stable in the dominant system, then the corresponding queue in the original system must be stable. Conversely, if for some $\lambda_{1}$ in the dominant system, the queue at the first node saturates, then it will not transmit dummy packets, and as long as the first user has a packet to transmit, the behavior of the dominant system is identical to that of the original system since dummy packet transmissions are eliminated as we approach the stability boundary. Therefore, the original and the dominant system are indistinguishable at the boundary points.

Remark 1. The stability region is a convex polyhedron when the following condition holds $\frac{\alpha_{1} \widehat{\alpha}_{2}}{\alpha_{1}^{*} P_{1}\{1\}}+\frac{\alpha_{2} \widehat{\alpha}_{2}}{\alpha_{2}^{*} P_{2 /\{2\}}} \geq 1$. When equality holds in the previous condition, see Fig. 2, the region is a triangle and coincides with the case of time-sharing. Convexity is an important property since it corresponds to the case when parallel concurrent transmissions are preferable to a time-sharing scheme. Additionally, convexity implies that if two rate pairs are stable, then any rate pair lying on the line segment joining those two rate pairs is also stable.

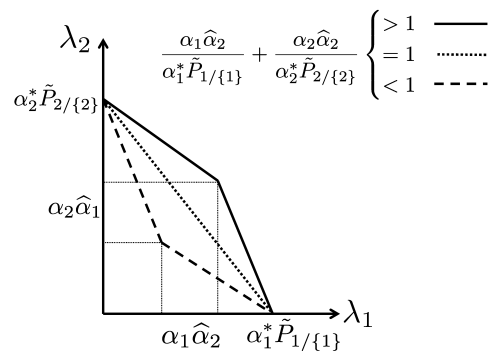

Fig. 2. The stability region described in Theorem III.1.

\section{Preparatory Analysis}

We now proceed with the investigation of the queueing delay at user nodes by providing the initial steps for the analysis. The key element is the derivation of the generating function of the joint stationary queue length distribution of the users. Denote by $N_{k, n}$ the number of packets at node $k, k=1,2$, at the beginning of slot $n$, and let $N_{k}=\lim _{n \rightarrow \infty} N_{k, n}$. Clearly, $Y_{n}=\left(N_{1, n}, N_{2, n}\right)$ is a discrete time Markov chain with state space $\{(i, j): i, j=0,1,2, \ldots\}$. The users' queues evolve as: $N_{k, n+1}=\left[N_{k, n}+\tilde{D}_{k, n}\right]^{+}+A_{k, n}, k=1,2$, where $\tilde{D}_{k, n}$ is the number of departures from user $k$ queue at time slot $n$, and $[x]^{+}=\max (0, x)$. Recall that $A_{k, n}$ is the number of packets arriving in user node $k, k=1,2$, during the time interval $(n, n+1]$. Let $H(x, y)=\lim _{n \rightarrow \infty} E\left(x^{N_{1, n}} y^{N_{2, n}}\right),|x| \leq 1$, $|y| \leq 1$ be the generating function of the joint stationary queue process. We consider independent geometrically distributed arrival processes, and thus, the generating function of the joint stationary distribution of the number of arrivals during $(n, n+1]$ is $D(x, y)=\lim _{n \rightarrow \infty} E\left(x^{A_{1, n}} y^{A_{2, n}}\right)=\left[\left(1+\lambda_{1}(1-\right.\right.$ $\left.x))\left(1+\lambda_{2}(1-y)\right)\right]^{-1},|x| \leq 1,|y| \leq 1$. Then,

$$
\begin{aligned}
R(x, y) H(x, y)= & A(x, y) H(x, 0)+B(x, y) H(0, y) \\
& +C(x, y) H(0,0),
\end{aligned}
$$

where,

$$
\begin{aligned}
R(x, y)= & D^{-1}(x, y)-1+\alpha_{1}\left(\bar{\alpha}_{2} P_{1 /\{1\}}+\alpha_{2} P_{1 /\{1,2\}}\right) \\
& \times\left(1-\frac{1}{x}\right)+\alpha_{2}\left(\bar{\alpha}_{1} P_{2 /\{2\}}+\alpha_{1} P_{2 /\{1,2\}}\right)\left(1-\frac{1}{y}\right) \\
& +\alpha_{1} \alpha_{2} P_{1,2 /\{1,2\}}\left(1-\frac{1}{x y}\right), \\
A(x, y)= & \alpha_{2}\left(\bar{\alpha}_{1} P_{2 /\{2\}}+\alpha_{1} P_{2 /\{1,2\}}\right)\left(1-\frac{1}{y}\right) \\
& +d_{1}\left(1-\frac{1}{x}\right)+\alpha_{1} \alpha_{2} P_{1,2 /\{1,2\}}\left(1-\frac{1}{x y}\right), \\
B(x, y)= & \alpha_{1}\left(\bar{\alpha}_{2} P_{1 /\{1\}}+\alpha_{2} P_{1 /\{1,2\}}\right)\left(1-\frac{1}{x}\right) \\
& +d_{2}\left(1-\frac{1}{y}\right)+\alpha_{1} \alpha_{2} P_{1,2 /\{1,2\}}\left(1-\frac{1}{x y}\right), \\
C(x, y)= & d_{2}\left(\frac{1}{y}-1\right)+d_{1}\left(\frac{1}{x}-1\right)+\alpha_{1} \alpha_{2} P_{1,2 /\{1,2\}}\left(\frac{1}{x y}-1\right) .
\end{aligned}
$$

For a detailed treatment see the longer version of this work in [22]. Our primary aim is to solve (5). Some interesting relations can be obtained directly from (5). Taking $y=1$, dividing by $x-1$ and taking $x \rightarrow 1$ in (5) and vice versa yield the following "conservation of flow" relations:

$$
\begin{aligned}
& \lambda_{1}=\alpha_{1} \widehat{\alpha}_{2}(1-H(0,1))-\widehat{d}_{1}(H(1,0)-H(0,0)), \\
& \lambda_{2}=\alpha_{2} \widehat{\alpha}_{1}(1-H(1,0))-\widehat{d}_{2}(H(0,1)-H(0,0)) .
\end{aligned}
$$

Based on (6) we distinguish the analysis in two cases, which differ both from the modeling and the technical point of view.

1) For $\frac{\alpha_{1} \widehat{\alpha}_{2}}{\alpha_{1}^{*} \tilde{P}_{1 /\{1\}}}+\frac{\alpha_{2} \widehat{\alpha}_{1}}{\alpha_{2}^{*} \tilde{P}_{2} /\{2\}}=1$, (6) yields $H(0,0)=1-$ $\frac{\lambda_{1}}{\alpha_{1}^{*} \tilde{P}_{1 /\{1\}}}-\frac{\lambda_{2}}{\alpha_{2}^{*} \tilde{P}_{2 /\{2\}}}=1-\rho$.

2) For $\frac{\alpha_{1} \widehat{\alpha}_{2}}{\alpha_{1}^{*} \bar{P}_{1 /\{1\}}}+\frac{\alpha_{2} \widehat{\alpha}_{1}}{\alpha_{2}^{*} P_{2 /\{2\}}} \neq 1$, (6) yields

$$
\begin{aligned}
& H(1,0)=\frac{\alpha_{1} \widehat{\alpha}_{2}\left(\lambda_{2}-\alpha_{2}^{*} \tilde{P}_{2 /\{2\}}\right)-\lambda_{1} \widehat{d}_{2}-\alpha_{1}^{*} \tilde{P}_{1 /\{1\}} \widehat{d}_{2} H(0,0)}{d_{1} d_{2}-\alpha_{1} \widehat{\alpha}_{2} \alpha_{2} \widehat{\alpha}_{1} \tilde{D}_{2} \widehat{x}_{1}}, \\
& H(0,1)=\frac{\alpha_{2} \widehat{\alpha}_{1}\left(\lambda_{1}-\alpha_{1}^{*} \tilde{P}_{1 /\{1\}}\right)-\lambda_{2} d_{1}-\alpha_{2}^{*} \tilde{P}_{2 /\{2\}} \widehat{d}_{1} H(0,0)}{d_{1} d_{2}-\alpha_{1} \widehat{\alpha}_{2} \alpha_{2} \widehat{\alpha}_{1}} .
\end{aligned}
$$

We now briefly summarize the technical steps that we have to follow in order to obtain expressions for the queueing delay at each user node. The key element is the solution of (5), which 
provides $H(x, y)$. However, we firstly have to derive $H(x, 0)$, $H(0, y), H(0,0)$. The basic steps are the following:

Step 1.: From (5), $H(x, 0)$ and $H(0, y)$ satisfy the Riemann-Hilbert $(\mathrm{RH})$ boundary value problems on closed curves. These curves are studied in Lemma V.1. The proof requires the investigation of the kernel $R(x, y)$, more details can be found in [22]. Clearly, $H(x, 0), H(0, y)$ they might have poles in the region bounded by the unit disc and these closed curves. With that in mind, we obtain the boundary conditions on these curves; see (9).

Step 2.: Next, we transform [17] these problems into the unit disc using conformal mappings; see SectionV.

Step 3.: We solve these new problems and we deduce an integral representation of the unknown functions; see (10).

\section{A RIEMANN-HiLbert BOUNDARY VALUE PROBLEM}

We consider first the kernel equation $R(x, y)=0$, and a subclass of MPR channels, the so called "capture" channels, under which, at most one user has a successful packet transmission even when many users transmit in that slot [23]. Thus, $P_{1,2 /\{1,2\}}=0$. In particular, $R(x, y)=a(x) y^{2}+b(x) y+$ $c(x)=\widehat{a}(y) x^{2}+\widehat{b}(y) x+\widehat{c}(y)$, where $a(x)=\lambda_{2} x\left(\lambda_{1}(x-1)-\right.$ $1), b(x)=x\left(\lambda+\lambda_{1} \lambda_{2}+\alpha_{1} \widehat{\alpha}_{2}+\alpha_{2} \widehat{\alpha}_{1}\right)-\alpha_{1} \widehat{\alpha}_{2}-\lambda_{1}\left(1+\lambda_{2}\right) x^{2}$, $c(x)=-\alpha_{2} \widehat{\alpha}_{1} x, \widehat{a}(y)=\lambda_{1} y\left(\lambda_{2}(y-1)-1\right), \widehat{b}(y)=y(\lambda+$ $\left.\lambda_{1} \lambda_{2}+\alpha_{1} \widehat{\alpha}_{2}+\alpha_{2} \widehat{\alpha}_{1}\right)-\alpha_{2} \widehat{\alpha}_{1}-\lambda_{2}\left(1+\lambda_{1}\right) y^{2}, \widehat{c}(y)=-\alpha_{1} \widehat{\alpha}_{2} y$.

Then, $R(x, y)=0$ defines the two-valued functions $X_{ \pm}(y)=\frac{-\widehat{b}(y) \pm \sqrt{D_{y}(y)}}{2 \widehat{a}(y)}, Y_{ \pm}(x)=\frac{-b(x) \pm \sqrt{D_{x}(x)}}{2 a(x)}$, where $D_{y}(y)=\widehat{b}(y)^{2}-4 \widehat{a}(y) \widehat{c}(y), D_{x}(x)=b(x)^{2}-4 a(x) c(x)$.

Denote by $X_{0}(y)$ (resp. $Y_{0}(x)$ ) the zero of $R(X(y), y)=0$ (resp. $R(x, Y(x))=0$ ) with the smallest modulus, and $X_{1}(y)$ (resp. $Y_{1}(x)$ ) the other one. Using Rouché's theorem, we can show that for $|y|=1, y \neq 1, R(x, y)=0$ has exactly one root $x=X_{0}(y)$ such that $\left|X_{0}(y)\right|<1$. Similarly, $y=Y_{0}(x)$, such that $\left|Y_{0}(x)\right|<1$, for $|x|=1$ (see Lemma IV.1 in [22]). Denote by $x_{i}$ (resp. $\left.y_{i}\right), i=1,2,3,4$, the zeros of $D_{x}(x)$ (resp. $D_{y}(y)$ ). In [22] we proved that $x_{i} \mathrm{~s}$ (resp $y_{i} \mathrm{~s}$ ) are such that $0<x_{1}<x_{2} \leq 1<x_{3}<x_{4}$ (resp. $0<y_{1}<y_{2} \leq 1<$ $\left.y_{3}<y_{4}\right)$, and $D_{x}(x)<0, x \in\left(x_{1}, x_{2}\right) \cup\left(x_{3}, x_{4}\right)$.

Lemma V.1. For $y \in\left[y_{1}, y_{2}\right], X(y)$ lies on a closed contour $\mathcal{M}$, which is symmetric with respect to the real line and defined by $|x|^{2}=m(\operatorname{Re}(x)), m(\delta)=$ $\frac{\alpha_{1} \widehat{\alpha}_{2}}{\lambda_{1}\left(1+\lambda_{2}-\lambda_{2} \zeta(\delta)\right)}$, and $|x|^{2} \leq \frac{\alpha_{1} \widehat{\alpha}_{2}}{\lambda_{1}\left(1+\lambda_{2}-\lambda_{2} y_{2}\right)}$, where, $\zeta(\delta)=$ $\frac{k(\delta)-\sqrt{k^{2}(\delta)-4 \alpha_{2} \widehat{\alpha}_{1}\left(\lambda_{2}\left(1+\lambda_{1}(1-2 \delta)\right)\right)}}{2 \lambda_{2}\left(1+\lambda_{1}(1-2 \delta)\right)}, \quad k(\delta):=\lambda+\lambda_{1} \lambda_{2}+$ $\alpha_{1} \widehat{\alpha}_{2}+\alpha_{2} \widehat{\alpha}_{1}-2 \lambda_{1}\left(1+\lambda_{2}\right) \delta$. Set $\beta_{0}:=\sqrt{\frac{\alpha_{1} \widehat{\alpha}_{2}}{\lambda_{1}\left(1+\lambda_{2}-\lambda_{2} y_{2}\right)}}$ is the extreme right point of $\mathcal{M}$.

A similar result also holds for $Y(x), x \in\left[x_{1}, x_{2}\right]$. We now proceed with the solution of (5). Due to space limitations, we only focus on the case, $\frac{\alpha_{1} \widehat{\alpha}_{2}}{\alpha_{1}^{*} \tilde{P}_{1 /\{1\}}}+\frac{\alpha_{2} \widehat{\alpha}_{1}}{\alpha_{2}^{*} \tilde{P}_{2 /\{2\}}} \neq 1$, more details can be found in the longer version of this work in [22]. Consider the transformation:

$$
\begin{aligned}
G(x) & :=H(x, 0)+\frac{\alpha_{1}^{*} \tilde{P}_{1 /\{1\}} d_{2} H(0,0)}{d_{1} d_{2}-\alpha_{1} \widehat{\alpha}_{2} \alpha_{2} \widehat{\alpha}_{1}}, \\
L(y) & :=H(0, y)+\frac{\alpha_{2}^{*} \tilde{P}_{2 /\{2\}} d_{1} H(0,0)}{d_{1} d_{2}-\alpha_{1} \widehat{\alpha}_{2} \alpha_{2} \widehat{\alpha}_{1}} .
\end{aligned}
$$

Then, for $y \in\left\{y \in \mathbb{C}:|y| \leq 1,\left|X_{0}(y)\right| \leq 1\right\}$, (5) becomes $A\left(X_{0}(y), y\right) G\left(X_{0}(y)\right)=-B\left(X_{0}(y), y\right) L(y)$. Using analytic continuation considerations, we have for $x \in \mathcal{M}$

$$
A\left(x, Y_{0}(x)\right) G(x)=-B\left(x, Y_{0}(x)\right) L\left(Y_{0}(x)\right) .
$$

Note that based on the values of the system parameters, $G(x)$ may has poles in $S_{x}:=G_{\mathcal{M}} \cap \bar{D}_{x}^{c}, \bar{D}_{x}^{c}=\{x \in \mathbb{C}:|x|>1\}$. These poles, if exist, coincide with the zeros of $A\left(x, Y_{0}(x)\right)$ in $S_{x}$. It can be shown that there exist at most one possible pole, say $\bar{x}$; see [22]. For $y \in\left[y_{1}, y_{2}\right], X_{0}(y)=x \in \mathcal{M}$ and $Y_{0}\left(X_{0}(y)\right)=y$. Taking into account the possible pole of $G(x)$ we have

$$
\begin{gathered}
\operatorname{Re}[i U(x) \tilde{G}(x)]=0, x \in \mathcal{M}, \\
U(x)=\frac{A\left(x, Y_{0}(x)\right)}{(x-\bar{x})^{r} B\left(x, Y_{0}(x)\right)}, \tilde{G}(x)=(x-\bar{x})^{r} G(x),
\end{gathered}
$$

where $r=0,1$, whether $\bar{x}$ is zero or not of $A\left(x, Y_{0}(x)\right)$ in $S_{x}$. Thus, $\tilde{G}(x)$ is regular for $x \in G_{\mathcal{M}}$, continuous for $x \in \mathcal{M} \cup$ $G_{\mathcal{M}}\left(G_{\mathcal{F}}\right.$ denotes the region bounded by the contour $\left.\mathcal{F}\right)$, and $U(x)$ is a non-vanishing function on $\mathcal{M}$. We must conformally transform the problem (9) from $\mathcal{M}$ to the unit circle $\mathcal{C}$, using the mapping $z=\gamma(x): G_{\mathcal{M}} \rightarrow G_{\mathcal{C}}$, and its inverse given by $x=\gamma_{0}(z): G_{\mathcal{C}} \rightarrow G_{\mathcal{M}}$. Then, the problem in (9) is reduced to the following: Find a function $F(z):=\tilde{G}\left(\gamma_{0}(z)\right)$, regular in $G_{\mathcal{C}}$, continuous in $G_{\mathcal{C}} \cup \mathcal{C}: \operatorname{Re}\left[i U\left(\gamma_{0}(z)\right) F(z)\right]=0, z \in \mathcal{C}$.

To proceed, we have to determine the index $\chi$ of (9); [17]. Following [20] (see Lemma IV.1 in [22]), we show that under ergodicity conditions, $\chi=0$, i.e., the problem in (9) has a unique solution given for $x \in G_{\mathcal{M}}$ by,

$$
\begin{aligned}
& H(x, 0)=\frac{\tilde{\lambda}_{1} d_{2}+\alpha_{1} \widehat{\alpha}_{2}\left(\alpha_{2}^{*} \tilde{P}_{2 /\{2\}}-\tilde{\lambda}_{2}\right)}{\left(\alpha_{1} \widehat{\alpha}_{2} \alpha_{2} \widehat{\alpha}_{1}-d_{1} d_{2}\right)} \\
& \times\left\{\exp \left[\frac{\gamma(x)-\gamma(1)}{2 i \pi} \int_{|t|=1} \frac{\log \{J(t)\}}{(t-\gamma(x))(t-\gamma(1))} d t\right]\right. \\
& \left.+\frac{\alpha_{1}^{*} \tilde{P}_{1 /\{1\}} d_{2}}{\alpha_{1} \widehat{\alpha}_{2} \alpha_{2}^{*} \tilde{P}_{2 /\{2\}}} \exp \left[\frac{-\gamma(1)}{2 i \pi} \int_{|t|=1} \frac{\log \{J(t)\}}{t(t-\gamma(1))} d t\right]\right\} .
\end{aligned}
$$

Similarly, we can determine $H(0, y)$ by solving another RH boundary value problem on the closed contour $\mathcal{L}$. Then, using (5) we uniquely obtain $H(x, y)$.

In addition, we need to determine the conformal mapping and its inverse. To proceed, we need a representation of $\mathcal{M}$ in polar coordinates, i.e., $\mathcal{M}=\{x: x=\rho(\phi) \exp (i \phi), \phi \in$ $[0,2 \pi]\}$. For further details see [17], [22]. Then, the mapping from $z \in G_{\mathcal{C}}$ to $x \in G_{\mathcal{M}}$ is uniquely determined by,

$$
\begin{aligned}
& \gamma_{0}(z)=z \exp \left[\frac{1}{2 \pi} \int_{0}^{2 \pi} \log \{\rho(\psi(\omega))\} \frac{e^{i \omega}+z}{e^{i \omega}-z} d \omega\right],|z|<1, \\
& \psi(\phi)=\phi-\int_{0}^{2 \pi} \log \{\rho(\psi(\omega))\} \cot \left(\frac{\omega-\phi}{2}\right) d \omega, 0 \leq \phi \leq 2 \pi,
\end{aligned}
$$

with $\psi(\phi)=2 \pi-\psi(2 \pi-\phi)$.

Denote by $H_{1}(x, y), H_{2}(x, y)$ the derivatives of $H(x, y)$ with respect to $x, y$ respectively. Then, the expected number of packets and the average delay at each user node in steady state are $M_{i}=H_{i}(1,1), \mathcal{D}_{i}=M_{i} / \lambda_{i}$. Using (5), (6),

$$
M_{1}=\frac{\lambda_{1}+d_{1} H_{1}(1,0)}{\alpha_{1} \widehat{\alpha}_{2}}, \quad M_{2}=\frac{\lambda_{2}+d_{2} H_{2}(0,1)}{\alpha_{2} \widehat{\alpha}_{1}} .
$$

We consider only $M_{1}, \mathcal{D}_{1}$, similarly we can obtain $M_{2}, \mathcal{D}_{2}$. Note that $H_{1}(1,0)$ can be obtained using (10),

$$
\begin{aligned}
H_{1}(1,0)= & \frac{\lambda_{1} d_{2}+\alpha_{1} \widehat{\alpha}_{2}\left(\alpha_{2}^{*} \tilde{P}_{2 /\{2\}}-\lambda_{2}\right)}{\alpha_{1} \widehat{\alpha}_{2} \alpha_{2} \widehat{\alpha}_{1}-d_{1} d_{2}} \\
& \times\left\{\frac{\gamma^{\prime}(1)}{2 \pi i} \int_{|t|=1} \frac{\log \{J(t)\}}{(t-\gamma(1))^{2}} d t+\frac{r}{1-\bar{x}} 1_{\{r=1\}}\right\} .
\end{aligned}
$$


Substitute in (11) to obtain $M_{1}$, and divide with $\lambda_{1}$, to obtain $\mathcal{D}_{1}$. For the calculation of $\gamma(1), \gamma^{\prime}(1)$, refer to [17], Ch. IV.1.

\section{THE SYMMETRICAL MODEL}

In this section, we consider the symmetrical model and we obtain closed form expressions for the average delay for the collision and the capture channel model, without explicitly computing the generating function for the stationary joint queue length distribution. Moreover, we provide upper and lower delay bounds for the MPR model. By symmetrical, we mean that $\alpha_{i}^{*}=\alpha^{*}, \alpha_{i}=\alpha, \lambda_{i}=\lambda, P_{i /\{i\}}=p, \tilde{P}_{i /\{i\}}=\tilde{p}$, $P_{i /\{1,2\}}=b, P_{1,2 /\{1,2\}}=c$. Due to the symmetry, we have $H_{1}(1,1)=H_{2}(1,1), H_{1}(1,0)=H_{2}(0,1)$. Thus, using (5),

$$
M_{1}=\frac{\lambda+\left(d+\alpha^{2} c\right) H_{1}(1,0)}{\alpha(p+\alpha(b+c-p))-\lambda} .
$$

Setting $x=y$ in (5), differentiating it with respect to $x$ at $x=1$, and using (6) we obtain

$$
2 M_{1}=\frac{2 \lambda-\lambda^{2}+\alpha^{2} c P\left(N_{1}>0, N_{2}>0\right)+2 H_{1}(1,0)\left(\alpha\left(p+\alpha(b-p)+d+2 \alpha^{2} c\right)\right)}{2(\alpha(p+\alpha(b+c-p))-\lambda)} .
$$

Using (12) and (13) we finally obtain $M_{1}=M_{2}=M$.

$$
M=\frac{\lambda\left[2\left(\alpha+\alpha^{2}(b+c-p)\right)+\lambda\left(d+\alpha^{2} c\right)\right]}{2 \alpha^{*} \tilde{p}(\alpha(p+\alpha(b+c-p))-\lambda)}-\frac{\alpha^{2} c\left(d+\alpha^{2} c\right) P\left(N_{1}>0, N_{2}>0\right)}{2 \alpha^{*} \tilde{p}(\alpha(p+\alpha(b+c-p))-\lambda)} .
$$

Using Little's law, the average delay in a node is given by

$$
\mathcal{D}_{1}=\mathcal{D}_{2}=\mathcal{D}=\frac{2\left(\alpha+\alpha^{2}(b+c-p)\right)+\lambda\left(d+\alpha^{2} c\right)}{2 \alpha^{*} \tilde{p}(\alpha(p+\alpha(b+c-p))-\lambda)}+\phi,
$$

where $\phi=-\frac{\alpha^{2} c\left(d+\alpha^{2} c\right) P\left(N_{1}>0, N_{2}>0\right)}{2 \lambda \alpha^{*} \tilde{p}(\alpha(p+\alpha(b+c-p))-\lambda)}$. Note that $\alpha(p+\alpha(b+$ $c-p))>\lambda$ due to the stability conditions.

If $c=0$, i.e., the "capture" model, the exact average delay in a node is given by (14) for $\phi=0$. When $c \neq 0$, i.e., strong MPR effect, we have two cases, (i) $d+\alpha^{2} c<0$, and $d+\alpha^{2} c>0$, which lead to the determination of upper/lower delay bounds. We focus only the former case, i.e., $\phi>0$ thus,

$$
\begin{aligned}
\mathcal{D}^{\text {low }} & =\frac{2\left(\alpha+\alpha^{2}(b+c-p)\right)+\lambda\left(d+\alpha^{2} c\right)}{2 \alpha^{*} \tilde{p}(\alpha(p+\alpha(b+c-p))-\lambda)}, \\
\mathcal{D}^{\text {up }} & =\mathcal{D}^{\text {low }}-\frac{\alpha^{2} c\left(d+\alpha^{2} c\right)}{2 \lambda \alpha^{*} \tilde{p}(\alpha(p+\alpha(b+c-p))-\lambda)} .
\end{aligned}
$$

\section{NUMERICAL RESUlTS}

In this section, we provide numerical results to validate the analysis presented earlier. We consider the case where the users have the same link characteristics and transmission probabilities to facilitate exposition clarity, so we will use the notation from Section VI.

In Figs. 3, 4, 5 the stability region for the collision, capture and MPR channel cases respectively are depicted where $p=$ $\tilde{p}=1, \alpha=0.6$ and various values of $\alpha^{*} \geq 0.6$. For the capture channel we have $b=0.4$ and $c=0$. For the MPR we have $b=0.4$ and $c=0.2$. In all three regions, the case where $\alpha^{*}=\alpha$ denotes the traditional random access scheme without adaptation. In all cases, we observe the profound advantage of the proposed adaptive scheme. As $\alpha^{*}$ increases, the stability region becomes broader, meaning that the system can sustain a stable behavior for larger values of the arrival rates.

The effect of the arrival rate $\lambda$ on the average delay is depicted in Fig. 6 for the collision, capture and the MPR models. We consider the case with $\alpha=0.6, p=0.9, \tilde{p}=1$.

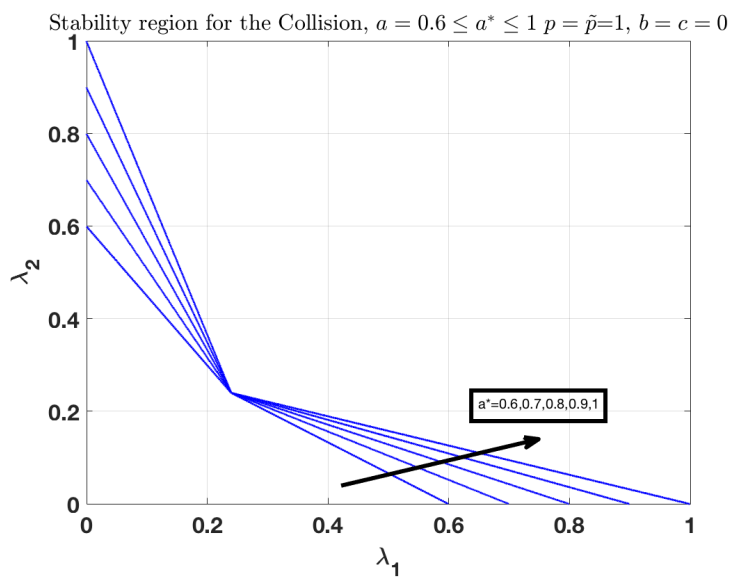

Fig. 3. The stability region for the collision channel.

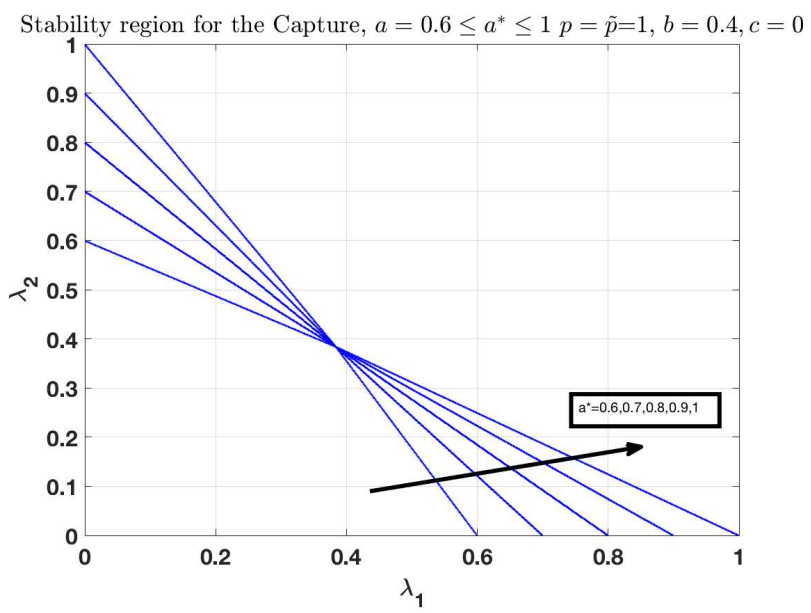

Fig. 4. The stability region for the capture channel with $b=0.4$ and $c=0$.

In addition, we consider three values for $\alpha^{*}=0.6,0.8,1$. The case $\alpha^{*}=0.6$ corresponds to the traditional random access scheme where the nodes do not adapt their transmission probabilities. The case $\alpha^{*}=1$ captures the scenario where a node transmits with probability 1 when the other node is empty. A first observation is that regarding the MPR channel model, the lower and the upper bounds appear to be close. As also expected, the average delay is lower for the MPR than the capture and the collision. Finite delay, or equivalently stability, can be sustained for larger values of $\lambda$ for the MPR case.

In Fig. 6, we also observe the effect of $\alpha^{*}$ on the average delay as $\lambda$ varies. As $\alpha^{*}$ increases, then average delay decreases, while the maximum arrival rate that can still maintain a finite delay is getting larger. This conclusion can be extracted from the set of values of the arrival rate that is depicted.

Clearly, by adapting the transmission characteristics of a node based on the queue state the other node would improve the system's performance.

\section{CONCLUSIONS}

In this work, we investigated the performance of an asymmetric two-user random access network with a queue-aware 


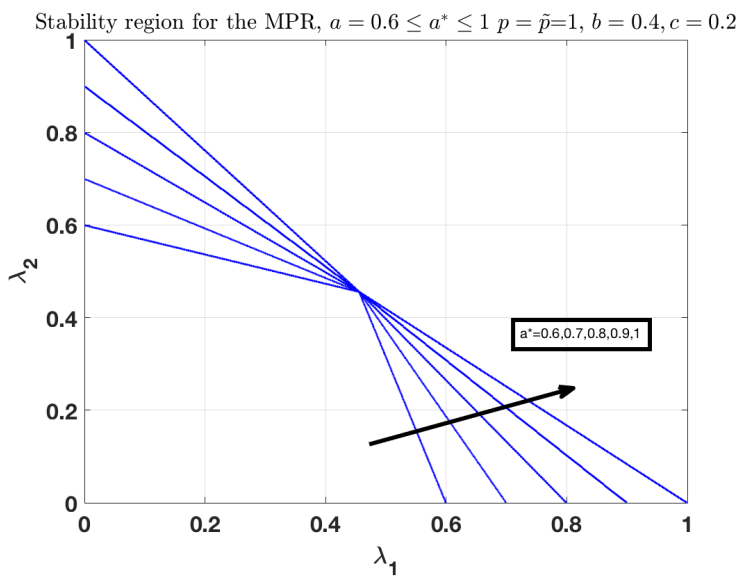

Fig. 5. The stability region for the MPR with $b=0.4$ and $c=0.2$.

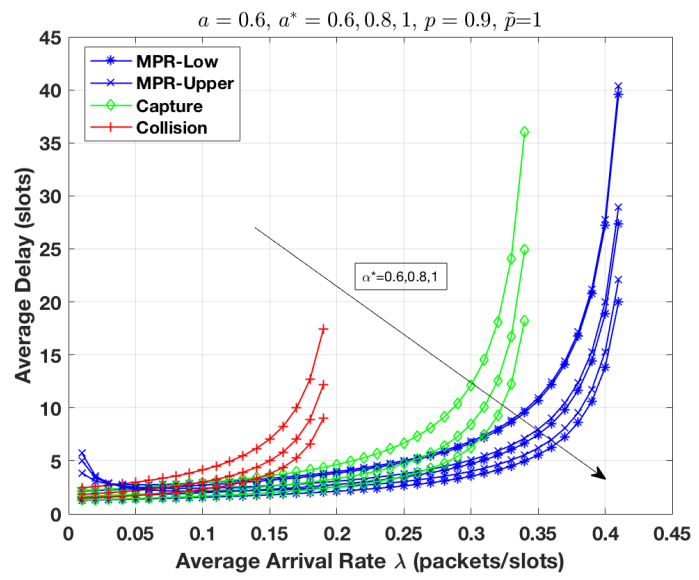

Fig. 6. Effect of $\lambda$ on the average delay for the collision, capture and the MPR channel models. The effect of $\alpha^{*}$ as $\lambda$ varies is also depicted. Recall that for the collision we have $b=c=0$, for the capture $b=0.4$ and $c=0$ and for the MPR $b=0.4$ and $c=0.2$.

transmission scheme. Based on the stochastic dominant systems we derived the stable throughput region. We performed a detailed mathematical analysis to investigate the queueing delay in terms of the solution of Riemann-Hilbert boundary value problem. Closed form expressions for the expected delay were derived for the symmetrical system with capture channel. For the symmetrical MPR model, we obtained bounds for the expected delay, and based on the numerical results the bounds are tight.

The current work serves as a building block towards the development of random-access networks with smart and selfaware nodes, which reconfigure their parameters based on their operational environment.

\section{REFERENCES}

[1] A. Laya, L. Alonso, and J. Alonso-Zarate, "Is the random access channel of LTE and LTE-A suitable for M2M communications? a survey of alternatives," IEEE Communications Surveys Tutorials, vol. 16, pp. 416, First 2014.

[2] A. Osseiran, F. Boccardi, V. Braun, K. Kusume, P. Marsch, M. Maternia, O. Queseth, M. Schellmann, H. Schotten, H. Taoka, H. Tullberg, M. A. Uusitalo, B. Timus, and M. Fallgren, "Scenarios for 5G mobile and wireless communications: the vision of the METIS project," IEEE Communications Magazine, vol. 52, pp. 26-35, May 2014.

[3] M. Koseoglu, "Lower bounds on the LTE-A average random access delay under massive M2M arrivals," IEEE Transactions on Communications, vol. 64, pp. 2104-2115, May 2016.

[4] J. B. Seo and V. C. M. Leung, "Performance modeling and stability of semi-persistent scheduling with initial random access in LTE," IEEE Transactions on Wireless Communications, vol. 11, pp. 4446-4456, December 2012.

[5] Z. Utkovski, O. Simeone, T. Dimitrova, and P. Popovski, "Random access in C-RAN for user activity detection with limited-capacity fronthaul," IEEE Signal Processing Letters, vol. 24, pp. 17-21, Jan 2017.

[6] H. Wang and T. Li, "Hybrid ALOHA: A novel MAC protocol," IEEE Transactions on Signal Processing, vol. 55, pp. 5821-5832, Dec 2007.

[7] Z. Utkovski, T. Eftimov, and P. Popovski, "Random access protocols with collision resolution in a noncoherent setting," IEEE Wireless Communications Letters, vol. 4, pp. 445-448, Aug 2015.

[8] R. Rao and A. Ephremides, "On the stability of interacting queues in a multiple-access system," IEEE Transactions on Information Theory, vol. 34, pp. 918-930, Sep 1988.

[9] W. Szpankowski, "Stability conditions for some distributed systems: buffered random access systems," Advances in Applied Probability, vol. 26, no. 2, pp. 498-515, 1994.

[10] W. Luo and A. Ephremides, "Stability of $\mathrm{N}$ interacting queues in random-access systems," IEEE Transactions on Information Theory, vol. 45, pp. 1579-1587, Jul 1999.

[11] V. Naware, G. Mergen, and L. Tong, "Stability and delay of finiteuser slotted ALOHA with multipacket reception," IEEE Transactions on Information Theory, vol. 51, pp. 2636-2656, July 2005.

[12] G. Papadimitriou, N. Pappas, A. Traganitis, and V. Angelakis, "Network-level performance evaluation of a two-relay cooperative random access wireless system," Computer Networks, vol. 88, pp. 187 201, 2015.

[13] M. Ploumidis, N. Pappas, and A. Traganitis, "Flow allocation for maximum throughput and bounded delay on multiple disjoint paths for random access wireless multihop networks," IEEE Transactions on Vehicular Technology, vol. 66, pp. 720-733, Jan 2017.

[14] P. Nain, "Analysis of a two-node aloha-network with infinite capacity buffers," in Int. Seminar on Computer Networking and Performance Evaluation, pp. 49-63, September 1985.

[15] A. B. Behroozi-Toosi and R. R. Rao, "Delay upper bounds for a finite user random-access system with bursty arrivals," IEEE Transactions on Communications, vol. 40, pp. 591-596, Mar 1992.

[16] L. Georgiadis, L. Merakos, and P. Papantoni-Kazakos, "A method for the delay analysis of random multiple-access algorithms whose delay process is regenerative," IEEE Journal on Selected Areas in Communications, vol. 5, pp. 1051-1062, Jul 1987.

[17] J. Cohen and O. Boxma, Boundary value problems in queueing systems analysis. Amsterdam, Netherlands: North Holland Publishing Company, 1983.

[18] I. Dimitriou, "A two class retrial system with coupled orbit queues," Prob. Engin. Infor. Sc., vol. 31, no. 2, pp. 139-179, 2017.

[19] I. Dimitriou, "A queueing model with two types of retrial customers and paired services," Ann. Oper. Res., vol. 238, no. 1, pp. 123-143, 2016.

[20] G. Fayolle, R. Iasnogorodski, and V. Malyshev, Random walks in the quarter-plane: Algebraic methods, boundary value problems and applications, volume 40 of Applications of Mathematics. SpringerVerlag, Berlin, 1999.

[21] N. Pappas, M. Kountouris, A. Ephremides, and A. Traganitis, "Relayassisted multiple access with full-duplex multi-packet reception," IEEE Transactions on Wireless Communications, vol. 14, pp. 3544-3558, July 2015.

[22] I. Dimitriou and N. Pappas, "Stable throughput and delay analysis of a random access network with queue-aware transmission," arXiv:1704.02902 [cs.IT], pp. 1-30, 2017.

[23] M. Zorzi and R. R. Rao, "Capture and retransmission control in mobile radio," IEEE Journal on Selected Areas in Communications, vol. 12, pp. 1289-1298, Oct 1994. 